\title{
Numerical Simulation and Experimental Validation of Small-rotor Aerodynamic Characteristics
}

\author{
Yao Lei ${ }^{1,}$, Y Yuxia $\mathrm{Ji}^{2, \mathrm{~b}}$ and Changwei Wang ${ }^{2, \mathrm{c}}$ \\ School of Mechanical Engineering and Automation, Fuzhou University, Fuzhou 350116, China \\ ayaolei@fzu.edu.cn, bfzu_yuxiaji@163.com, c wangchangwei789@163.com
}

\begin{abstract}
Keywords: single-rotor, boundary conditions, computational fluid dynamics method, aerodynamic characteristics, experimental research.
\end{abstract}

\begin{abstract}
Aerodynamic characteristics of small-rotor were studied based on the finite volume method. In an attempt to study its performance, hovering flow field of the single-rotor was established, meanwhile, the boundary conditions were set up. In addition, flow field was analyzed numerically by using computational fluid dynamics method (CFD) which aimed at obtaining the distribution of surface pressure, the diagram of velocity vector and the flow chart of flow field. Simultaneously, the coefficients of lift and drag were acquired by data processing. In the analysis of the distribution features got from the numerical simulation of flow field, aerodynamic characteristics of the rotor were revealed generally. Equally important, experimental research and hover test stand were also designed to measure the actual thrust and power consumption of the system. Compared the results of numerical simulation with experimental verification, the speed range of higher lift-to-drag ratio and lower power consumption of a single-rotor were obtained.
\end{abstract}

\section{Introduction}

Small-rotor aircraft has the ability to take off and land vertically. Its hover performance and forward flight with high-speed are also excellent. The characteristics of small-size, light-weight and simple-structure are also its advantages. It's easy to control and fly freely within minimal takeoff and landing zones. These characteristics make it attractive for the applications of monitoring, aerial shots, exploration, rescue, etc. Rotor is the basis of the study on the aerodynamic performance of small-rotor aircraft. When it flies through the air, the aerodynamic force is mainly produced by rotor. And the numbers of rotor blades, also the shape, radius of rotor and blade width, besides, the angle of attack, rotate speed and so on all have effects on the aerodynamic performance of the small-rotor aircraft [1].Many scholars at home and abroad have carried on the related research on the factors which can influence aerodynamic performance of the rotor. Q J. Gao, et al performed aerodynamic simulation for comparing different camber, thickness and angle of attack of rotor in a low Reynolds number [2]. Y. Bai, et al attempted to use the airfoil profile of Eppler387 to analysis the lift-to-drag characteristics [3]. L Q. Ji, et al studied on the aerodynamic characteristics of isolated rotor and coaxial rotor [4].Y M. Deng, et al made efforts on the relationship with aerodynamic interference and advance ratio between the upper and lower rotor, when the rotors hover and fly forward [5]. Thomas J. Muller, et al describes the influences of the Reynolds number and aspect ratio on the design and aerodynamic performance of aircraft [6].Y. Lei, et al did research on the influences of the configuration and wind speed on aerodynamic characteristics of coaxial rotor [7]. L H. Yang analyzed the aerodynamic characteristics and put forward the design scheme of six-rotor large loads UAV [8].V. K. Lakshminarayan explored the effects of speed and spacing of the rotors on the performance of micro coaxial aircraft [9]. Moble Benedict compared the hovering characteristics under different airfoil profile of miniature helicopter [10].Stephen D.P rior performed experiments on how the size and distance of the rotor exercise great influence on the drag of small unmanned aerial vehicle (UAV) [11]. Caitlin Powers considered the influence of rotor speed and wind speed on the four-rotor aircraft's lift [12].B.R. Hein, et al reflected on the hovering performance of MAV in low Reynolds number [13].H. Won Kim, et al did aerodynamic modeling from an isolated rotor to a complete aircraft of coaxial helicopter [14]. 
The speed of the isolated rotor can directly affect the aircraft aerodynamic characteristics, such as lift, drag, power consumption, etc. Take a single rotor as the research object and use the CFD method to do numerical simulation for hovering flow field of the isolated rotor. Rotor is made of carbon fiber materials with light weight and compact model. In the process of numerical simulation, as an isolated rotor, it is assumed that flow fluid surround every profile of the rotor is the same. The effects of any solid wall except the rotor itself were shut out. It is only considered that the rotor motions in static fluid or uniform flow surrounds the airfoil profile [15].In numerical simulation, the speed range of the rotor is $909 \mathrm{r} / \mathrm{min}$ to $2182 \mathrm{r} / \mathrm{min}$. In order to confirm the rotor's properties of lift and drag at different rotational speed, the pressure distribution, vector diagram, streamlines of flow field are also as important factors to analyze aerodynamic characteristics and distribution features of flow field. Finally, as a validation test research, the lift and power consumption of the rotor at different rotational speed was measured. Compared with the results of numerical simulation and experiments, the speed range of high lift-to-drag ratio and low consumption of an isolated rotor was obtained.

\section{Theoretical Calculation}

Definitions of the formula [17] for lift and drag are as follows:

$$
\begin{aligned}
& L=\frac{1}{2} \rho C_{l} \omega^{2} \\
& D=\frac{1}{2} \rho C_{d} \omega^{2}
\end{aligned}
$$

Where $L$ is the single rotor's lift, $D$ the single rotor's drag, $\rho$ the air density and the value is 1.225 $\mathrm{kg} / \mathrm{m}^{3}, C_{l}$ the coefficient of lift, $C_{d}$ the coefficient of lift and $\omega$ is the angular velocity of rotor.

Lift-to-drag ratio is an important characteristic to measure aerodynamic performance of rotor. Lift and drag coefficient can be calculated by formula and numerical simulation. The formula [16] of $C_{l}$ and $C_{d}$ are defined as follows:

$$
\begin{aligned}
C_{l} & =\frac{F_{y}}{0.5 \rho U_{r e f}^{2} S_{r e f}} \\
C_{d} & =\frac{F_{x}}{0.5 \rho U_{r e f}^{2} S_{r e f}}
\end{aligned}
$$

Where $U_{r e f}$ is the reference velocity or flow velocity and $S_{r e f}$ is the area for reference or the rotor's projection area.

Depending on the values of rotor's lift and power consumption which was measured by experiment, thrust coefficient $C_{T}$ and power coefficient $C_{P}$ of the rotor can be written as [7].

$$
\begin{aligned}
& C_{T}=\frac{T}{\rho A \Omega^{2} R^{2} \Delta} \\
& C_{P}=\frac{P}{\rho A \Omega^{3} R^{3} \Delta} \\
& \Delta=\frac{p \times 288.15}{1013 \times(t+273.15)}
\end{aligned}
$$

Where $\Delta$ is the ratio of local atmospheric density and standard atmospheric density of the sea level, $p$ the atmospheric pressure, $t$ the Celsius temperature, $T$ the thrust of the rotor, $P$ the power of the rotor, $A$ the rotor disk area, $\Omega$ the angular velocity of blade rotation and $R$ the radius of the rotor.

\section{Numerical Simulation}

\subsection{Basic Parameters of Rotor.}

The rotor radius and the average blade chord of the rotor are $200 \mathrm{~mm}$ and $35 \mathrm{~mm}$, respectively. In addition, the rotor has two blades and the rating speed is $900 \mathrm{r} / \mathrm{min} \sim 2200 \mathrm{r} / \mathrm{min}$ (Table 1). 
Table 1 Basic parameters of rotor

\begin{tabular}{cccc}
\hline Radius /mm & Number of blades & Rating speed/RPM & Chord length/mm \\
\hline 200 & 2 & $900 \sim 2200$ & 35 \\
\hline
\end{tabular}

\subsection{Process of the Simulation.}

First, the flow field was designed, and grid was also divided. Furthermore, entering the software of FLUENT and checking the skewness of the grid were to ensure that there is no negative grid. On the one hand, the format and control equation were selected. On the other hand, the fluid properties were defined and the boundary conditions were set at the same time. At last, the reference values were input and initialized the flow field after iteration calculation. It is of great significance that we need judge the condition of convergence after save calculation results, then, enter the CFD-Post. Setup of the boundary conditions for the whole flow field is shown as follows:

a) Because of the mach number of blade tip, it is unnecessary to consider the compressibility. We ought to choose the solver called Pressure-based which suitable for processing model of incompressible flow.

b) It is suitable for the simulation of flow field in terms of small rotor with no need for dense grid, and the choice of viscous model is Spalart-Allmaras.

c) It is obvious that Wall is significantly fit to limit the regions of fluid and solid [18],hence,the boundary condition of Wall is appropriate. The rotor is set as absolute motion, while the relative speed of fluid boundary is set at zero.

d) Setting the reference values is crucial for the results, such as area of the rotor, chord length of the airfoil, air density, viscosity and pressure, etc.

e) The scheme of pressure-velocity coupling is SIMPLE, and spatial discretization of pressure is Standard. The choice of gradient is Least Squares Cell Based, while the momentum and modified turbulent viscosity choose First Order Upwind.

f) On the one hand, in an attempt to set up the monitors of residual,lift, drag and monitoring the condition of convergence is the basis of the output of data. On the other hand, it is required to enter CFD-Post for processing the pressure diagram and flow chart of rotor.

The setup process of flow field of is shown in Fig.1:

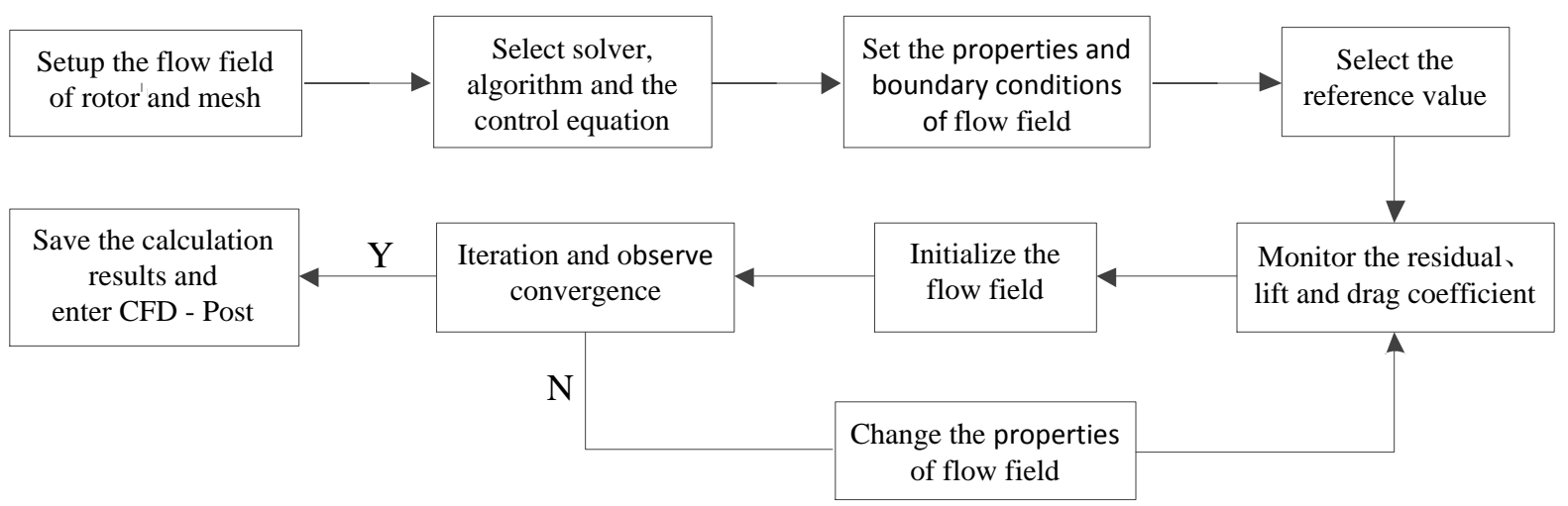

Fig. 1 Setup of the flow field

Numerical simulation mainly is to figure out the characteristics of lift-to-drag, the vector distribution, pressure distribution of rotor's surface and streamlines of flow field at different rotational speed. Experiments were performed to make measurements of thrust and power consumption. Experiments were conducted by changing the rotational speed (RPM) roughly arrange from 909 RMP to $2200 \mathrm{RPM}$, and the specific selected speed is $909 \mathrm{r} / \mathrm{min}, 1008 \mathrm{r} / \mathrm{min}, 1185 \mathrm{r} / \mathrm{min}$ and $1359 \mathrm{r} / \mathrm{min}, 1491 \mathrm{r} / \mathrm{min}$ and $1604 \mathrm{r} / \mathrm{min}, 1707 \mathrm{r} / \mathrm{min}$ and $1805 \mathrm{r} / \mathrm{min}, 1912 \mathrm{r} / \mathrm{min}$ and $2008 \mathrm{r} / \mathrm{min}$, $2103 \mathrm{r} / \mathrm{min}$ and $2182 \mathrm{r} / \mathrm{min}$. Based on all the speed which varies from each other, Numerical simulation and experimental measurements will get different results. 


\subsection{Analysis of Simulation Result.}

The pressure distribution of upper and lower surface in Fig. 2 shows that the red area which locates at the blade tip of rotor represents the highest pressure. Blade tip with a little dark green and blue area indicates that there is larger area of negative pressure. Due to the rotor rotation, the fluid which locates at the lower surface was sent around to the trailing edge point of upper surface, and formed the flow more than 180 degrees. The speed of the trailing edge point will reach infinity. As a consequence, the upper surface near the trailing edge point generated significant adverse pressure gradient. Pressure on the upper surface of the blade tip is smaller than the lower surface, and the closer distance from blade tip, the more pressure is. On the whole, the total pressure of the lower surface is greater than the upper surface. According to the principle of continuity and Bernoulli's principle, raised sections of airfoil's upper surface result in thinner stream. In other words, cross-sectional area of the stream decreases, while the velocity of air increases, that is, pressure decreasing. However, relatively flat the lower surface of airfoil is, rarely the stream change, which is means the pressure basically remain unchanged. Therefore, pressure difference generated from the upper and lower surface of airfoil, which created aerodynamic lift for the rise of rotor [15].

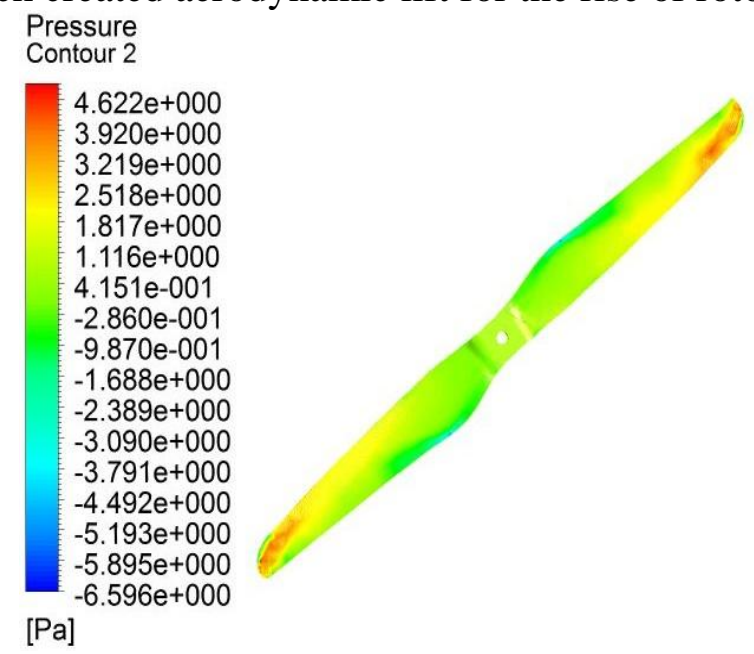

(a) Upper surface

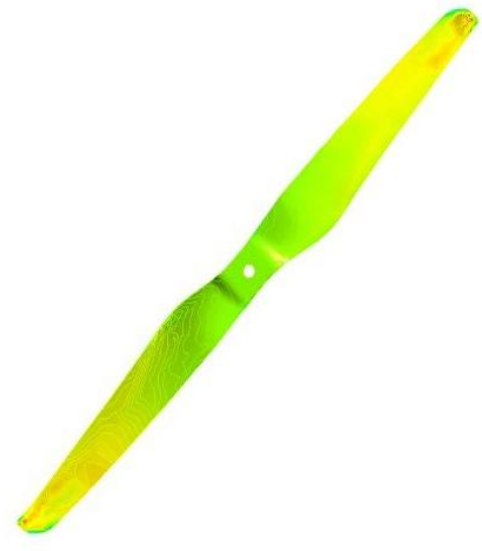

(b) Lower surface

Fig. 2 Pressure distribution of rotor

Velocity vector diagram of blade tip is showed in Fig.3. It was obvious that the tip vortex were produced under effect of rotor spinning. Due to the rotor started to accelerate gradually, the boundary layer unable to bear great adverse pressure gradient and immediately separated from the surface, thus formed whirlpools [15].By observing the pressure of the rotor's surface, it is obvious that the place of pressure gradient which is maximal from where escape the blade tip vortex, and the existence of blade tip vortex will reduce the efficiency of the rotor.

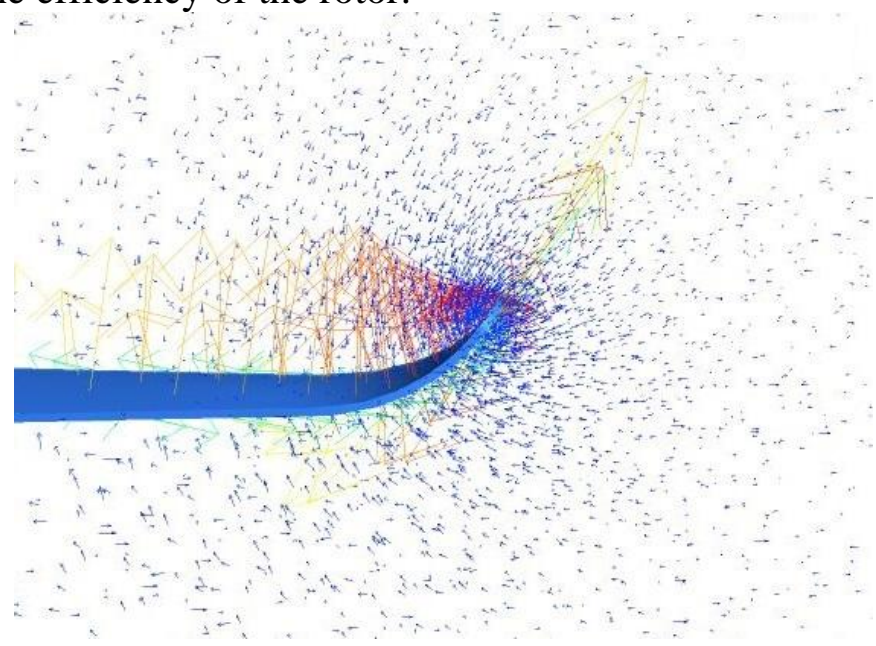

Fig. 3 Vector diagram of blade tip

Two views shows in Fig.4 is the streamlines distribution of flow field. It is indicated that radial distribution of streamlines are relatively even, and axial flow is spiraling. 


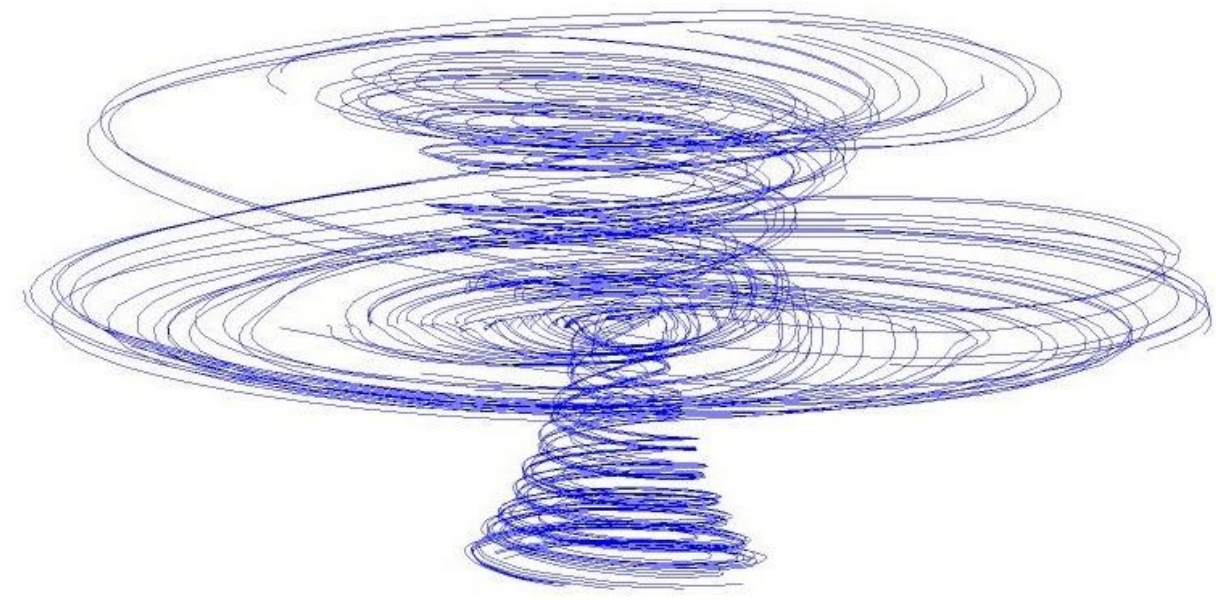

Fig. 4 Streamlines of flow field

Shown in Fig.5 are the coefficients of lift and drag which change with the rotating speed. It can be observed that lift coefficient and drag coefficient are rising steadily with the increase of rotational speed by the curve we can see from the picture. A conclusion can be deduced that increasing speed does not necessarily increase the lift to drag ratio. However, it will be taken more energy if the rotational speed increases. Therefore, it is essential that we should consider the relationships of speed, lift-to-drag ratio and power consumption, and determine the optimal solution.
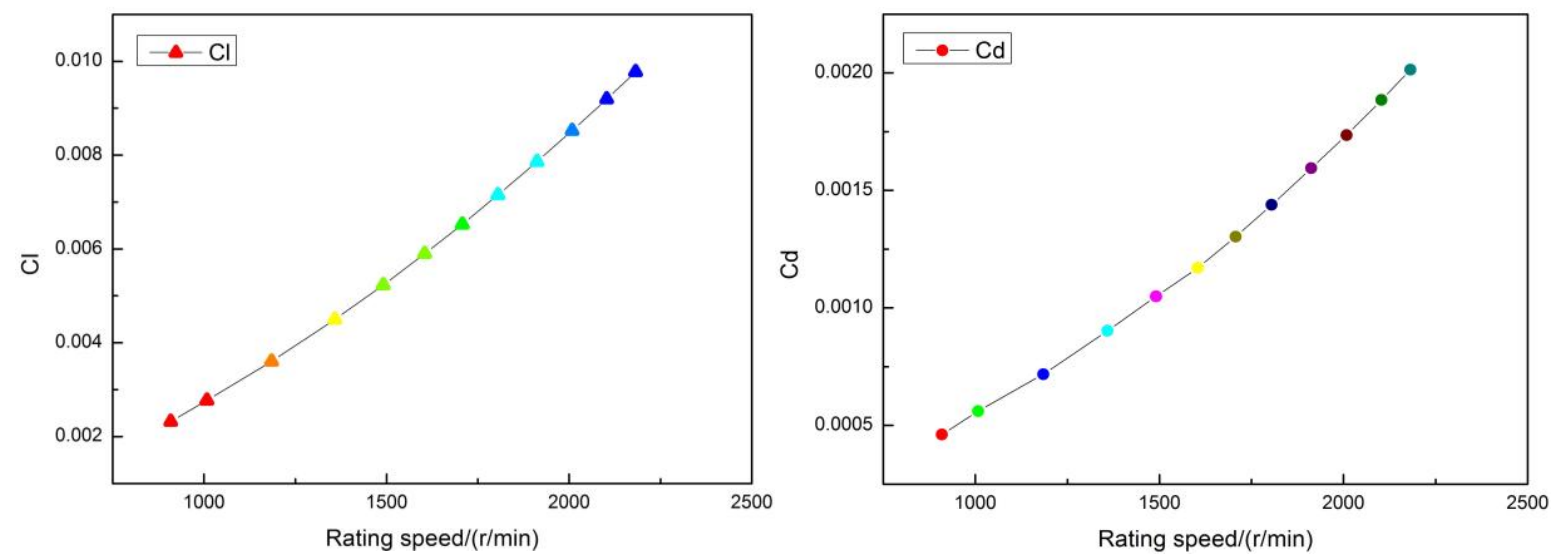

Fig. 5 Coefficients of lift and drag change with rating speed

\section{Experimental investigations}

\subsection{Design of test system.}

Testing equipment mainly includes the dynamic system, operating system, measurement system, bearing, which provide measurements of the rotor speed, thrust and power consumption.

(1) Power system

Power system is used for dynamics of rotor rotation, of which the equipment are respectively the dc power supply with the specifications of ace lithium polymer batteries, dc brushless motor with the specifications of HK-3020, electronic commutator and electronic governor with the specifications of Castle TALON35.There is no commutator brush in the brushless motor, therefore, an electronic commutator need to be added. It is able to keep the motor rotation. The speed of motor is often regulated by electronic governor which can be divided into brush electronic governor and brushless electronic governor [8].

(2) Operating system

The function of operating system is to control the rotor speed by changing the PWM duty ratios of control pulse to regulate average dc voltage which was input in the brushless dc motor (BLDCM), so as to realize speed regulation [16]. 
(3) Measurement system

The rotor speed, thrust and power consumption were measured by measurement system, and the equipment consisted of reflective sheeting, non-contact photoelectric speedometer with the specifications of VC6236P, force sensor and power supply of sensor with the specifications of MS3010D.With respect to speed measurement, the light was emitted by speedometer to measure the speed of the rotor after the reflective sheeting was installed on the motor shaft. Force sensor which was installed on the bracket has capabilities to convert the value of thrust to electrical signal output, while the power consumption is calculated by the current and voltage of the motor.

(4) Bearing

Main function of bearing is to support drive system of the rotor, of which the height is $1.3 \mathrm{~m}$ from the ground for the rotor to avoid the influence of ground effect on rotor aerodynamic characteristics.

\subsection{Sketch of Experimental Device.}

On the basis of numerical simulation, test stand was built to detect the aerodynamic characteristics of the hovering single rotor [19-20], such as thrust, rotate speed and power consumption, etc. Measured data was gathered into the DAQ Card (data acquisition card) and input into the computer. It is convenient for the data of observation, contrast and disposal. The sketch of the test stand is shown in Fig.6.

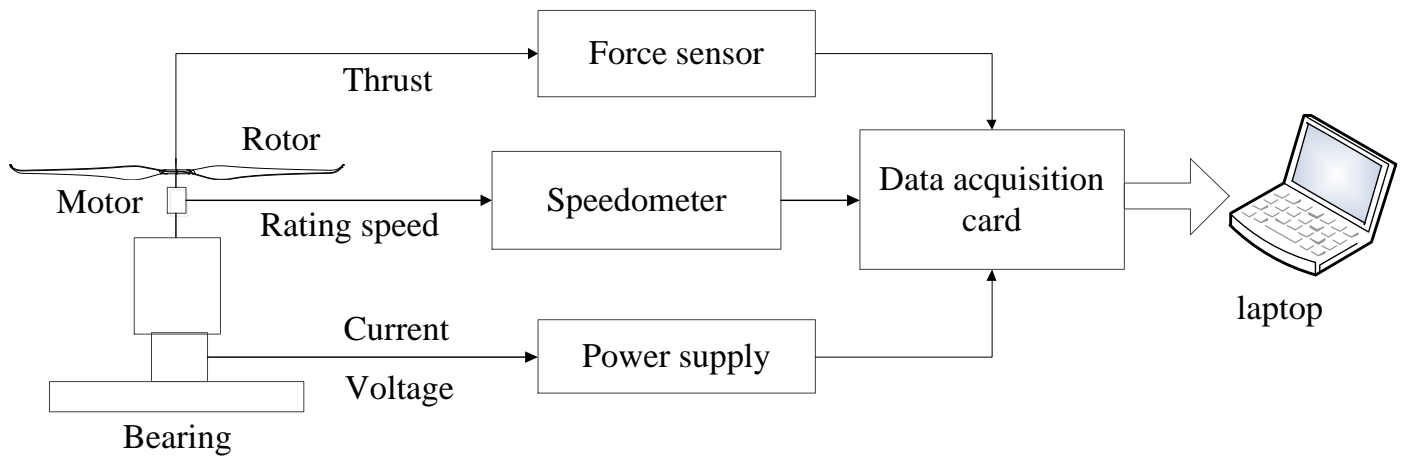

\subsection{Analysis of Test Result.}

Fig. 6 Experimental setup

As shown in Fig.7, it is obvious that thrust and power consumption are proportional to the rotating speed. The increase of thrust along with power consumption means that the more thrust rotor will get, the more energy will consume.
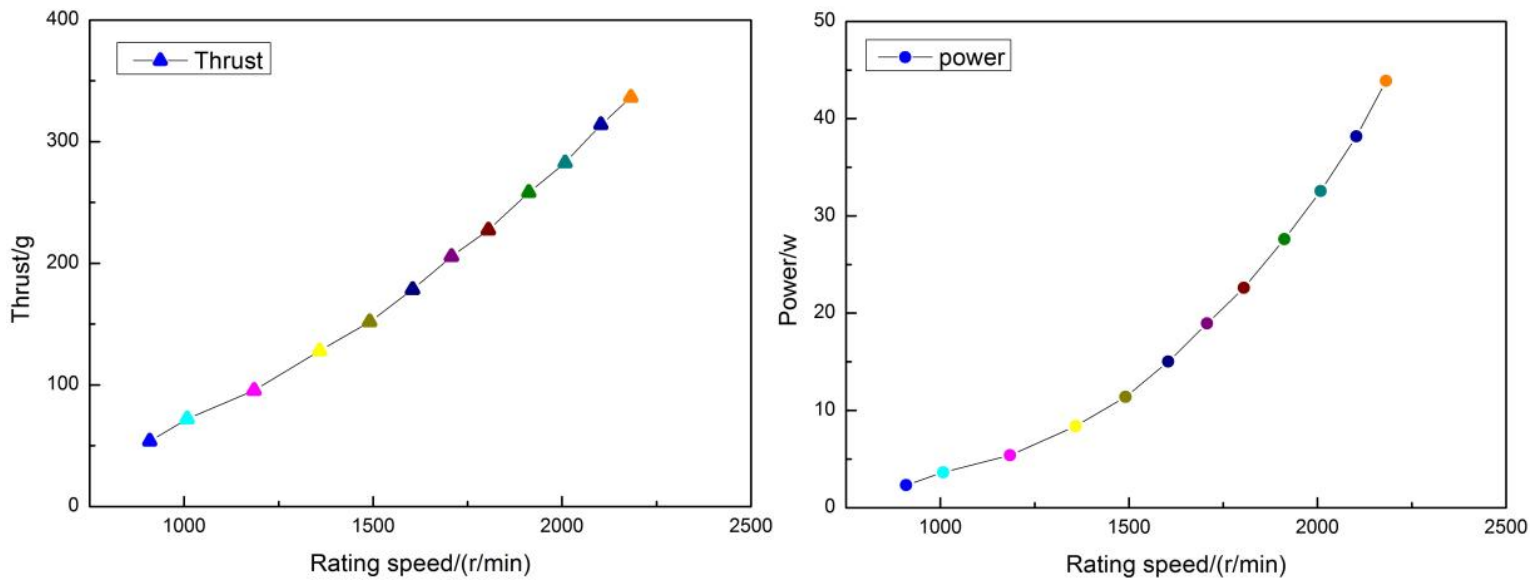

Fig. 7 Thrust and power change with rating speed

Thrust and power consumption measured by experiment were brought into the formula (6) and (7), and the coefficient of thrust and power can be calculated. Coefficients of thrust and power are shown in Fig.8. Due to the actual measurement error, there is decrease of thrust coefficient once in a while when the rotating speed increases, however, the general trend of which is increasing. Likewise, 
power coefficient at different rotating speed has the same rule to thrust coefficient. It was obvious that thrust coefficient began to stabilize, when the rotating speed at $1650 \mathrm{r} / \mathrm{min}$ to $2182 \mathrm{r} / \mathrm{min}$.

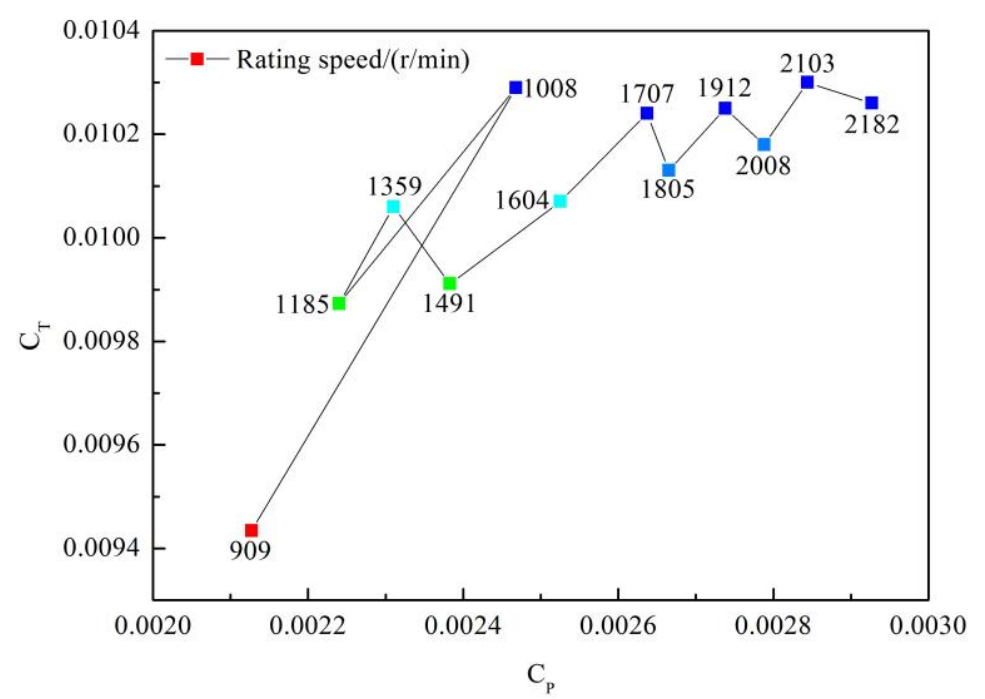

Fig. 8 Coefficients of thrust and power change with rating speed

It can be clearly obtained that thrust coefficient of average error is less than $0.5 \%$ when the rotating speed at $1650 \mathrm{r} / \mathrm{min}$ to $1664 \mathrm{r} / \mathrm{min}$. However, the power consumption increases with the speed increasing. On the premise of obtaining thrust coefficient which is stable and larger, the rotating speed of rotor at $1650 \mathrm{r} / \mathrm{min}$ to $1664 \mathrm{r} / \mathrm{min}$ with larger lift-to-drag ratio and less power consumption were chosen.

\section{Conclusion}

Small-rotor with light weight and small size can realize all kinds of flight attitude, and it adapts to complex terrain well. With broad and profound prospects, it was applied in monitoring, aerial shots, exploration, rescue, etc. In the meantime, differences in blade geometries, rotational speed, Reynolds number and angle of attack affect the inflow distribution, making it very difficult to draw a general conclusion about the aerodynamic performance of a small-rotor. Two parts were studied in this paper, one is numerical simulation, and the other is experimental study. It is crucial for the former to analyze the pressure of rotor's surfaces, the characteristics of hovering flow field and the coefficients of thrust and power. For the latter, four parts of experiment system were designed to measure the actual thrust and power consumption. Combined with the numerical simulation and experimental study, the following are the specific conclusions drawn on the present study on small-rotor:

(1)There is maximal pressure of blade tip where exists negative pressure. Pressure on the upper surface of the blade tip is smaller than the lower surface, and the closer distance from blade tip, the more pressure is. In addition, pressure difference generated from the upper and lower surface of airfoil, which created aerodynamic lift for the rise of rotor. It is obvious that the place of pressure gradient which is maximal from where escape the blade tip vortex, and the existence of blade tip vortex will reduce the efficiency of the rotor and affect the performance of the rotor.

(2)To observe the streamlines distribution of flow field, it is easily found that radial distribution of streamlines are relatively even, and axial flow is spiraling.

(3)Results of numerical simulation show that coefficients of lift and drag are rising steadily with the increase of rotational speed, however, increasing speed does not necessarily increase the lift to drag ratio.

(4)Results of experimental research show that thrust and power consumption are proportional to the rotating speed and the increase of thrust goes along with power consumption. Due to the actual measurement error, there is fluctuation of the values for thrust coefficient and power coefficient, but the general trend is increasing. 
(5)Thrust coefficient reached maximum and began to stabilize, when the rotating speed at 1650 $\mathrm{r} / \mathrm{min}$ to $2182 \mathrm{r} / \mathrm{min}$. However, the power consumption increases with the speed increasing. To compare the results of numerical simulation and experimental research, we can find that on the premise of obtaining thrust coefficient which is stable and larger, the rotating speed of rotor at 1650 $\mathrm{r} / \mathrm{min}$ to $1664 \mathrm{r} / \mathrm{min}$ with larger lift-to-drag ratio and less power consumption were desired.

\section{Acknowledgements}

This project is supported by National Natural Science Foundation of China (Grant No. 51505087) and The Education Department of Fujian Province (Grant No. JA15054).

\section{References}

[1]. J Z. Su, J P. Qian, W P. Huang, et al. Impact analysis of suspension bullets rotors structure parameters to the aerodynamic force [J]. Ordnance Industry Automation. Vol. 34 (2015) No. 6, p. 20-24.

[2]. Q J. Gao, Y. Bai, Q. Sun, et al. Design and aerodynamic simulation of low Reynolds number rotor airfoil [J]. Optics and Precision Engineering. Vol. 23 (2015) No. 2, p. 511-519.

[3]. Y. Bai, P. Cao, Q J. Gao, et al. Characterization of a multi rotor micro air vehicle and simulation of airfoil aerodynamics at low Reynolds-number [J].ACTA AERODYNAMICA. Vol. 29 (2011) No. 3, p. 325-329.

[4]. L Q. Ji, Q H. Zhu, J B. Li. Experimental investigation on aerodynamic characteristics of auto-rotating coaxial twin-rotor and single rotor [J]. Journal of Experiments in Fluid Mechanics. Vol. 27 (2013) No. 5, p.7-10.

[5]. Y M. Deng, R. Tao, J Z. Hu. Experimental investigation of the aerodynamic interaction between upper and lower rotors of a coaxial helicopter [J].ACTA AERONAUTICA ET ASTRONAUT ICA SINICA. Vol. 24 (2003) No. 1, p. 10-14.

[6]. Thomas J. Muller, James D Delaurier. Aerodynamics of small vehicles [J]. ADVANCES IN MECHANICS. Vol. 34 (2004) No. 2, p. 270-279.

[7]. Y. Lei, Y. Bai, Z. Xu. An experimental investigation on aerodynamic performance of a coaxial rotor system with different rotor spacing and wind speed [J]. Experimental Thermal and Fluid Science. Vol. 44 (2013) No. C, p. 779-785.

[8]. H L. Yang. Aerodynamic characteristics numerical simulation and optimization research of tandem six-rotor large loads UAV [D]. [Ph. D. Dissertation], School of mechanical science and engineering, Jilin university, China 2015. p. 1.

[9]. V. K. Lakshminarayan. Computational investigation of Micro-scale coaxial rotor aerodynamics in hover [D]. [Ph. D. Dissertation], Department of Aerospace Engineering, University of Maryland, America 2009. p. 1.

[10]. Moble Benedict, Justin Winslow, Zohaib Hasnain, et al. Experimental investigation of micro air vehicle scale helicopter rotor in hover [C]. American Helicopter Society Annual Forum. Montreal, Quebec, Canada, May 20-22, 2014, p. 231-255.

[11]. Stephen D. Prior. Reviewing and investigating the use of coaxial rotor systems in small UAVs [J]. International Journal of Micro Air Vehicle. Vol. 2 (2010) No. 1, p. 1-16.

[12]. Caitlin Powers, Daniel Mellinger, Aleksandr Kushleyev, et al. Influence of aerodynamics and proximity effects in quadrotor flight [J]. Experimental Robotics. Vol. 88 (2013) p. 289-302. 
[13]. B. R. Hein, I. Chopra. Hover performance of a micro air vehicle: rotors at low Reynolds number [C]. Proceedings of the American Helicopter Society International Specialists' Meeting on Unmanned Rotorcraft, Chandler, July 1, 2007, p. 254-262.

[14]. H. Won Kim, R. E. Brown. Modeling the aerodynamics of coaxial helicopters-from an isolated rotor to a complete aircraft [C]. EKC2008 Proceedings of the EU-Korea Conference on Science and Technology. Germany, 2008, p.45-59.

[15]. Q. Fu, G. Wei, H. Guan, et al. Advanced fluid mechanics [M].Nanjing: Southeast university press, 2014, p.36-47.

[16]. Y. Lei. Aerodynamics of a hex-rotor SUAV: numerical simulation and experimental study [D]. [Ph. D. Dissertation], Changchun Institute of Optics, Fine Mechanics and Physics Chinese Academy of Sciences, China 2013, p. 49.

[17]. S H. Qi, S J. Liu, W M. Zhang, et al. Analysis of aerodynamic and vibration characteristics of a micro quad-rotor helicopter with low Reynolds number [J]. Noise and Vibration Control .Vol. 33 (2013) No. 5, p. 53-57.

[18]. Y. Yu. Fluent introductory and advanced tutorial [M]. Beijing: BEIJING INSTITUTE OF TECHNOLOGY PRESS, 2008, p. 252-253.

[19]. F. Bohorquez. Rotor hover performance and system design of an efficient coaxial rotary wing micro air vehicle [D]. [Ph.D. dissertation], Department of Aerospace Engineering, University of Maryland, America 2007, p. 1.

[20]. Arnaud Koehl, Hugues Rafaralahy, Mohamed Boutayeb, et al. Aerodynamic modelling and experimental identification of a coaxial-Rotor UAV [J]. J Intell Robot Syst. Vol. 68 (2012) No. 1, p. 53-68. 